\title{
Characterization of a Multi-Electrode bulk-SOA for Low NF In-line Amplification in Passive Optical Networks
}

\author{
Kevin Carney, Sylwester Latkowski*, Ramón Maldonado-Basilio, and Pascal Landais \\ Research Institute for Networks and Communications Engineering (RINCE) \\ School of Electronic Engineering, Dublin City University, Glasnevin, Dublin 9, Ireland \\ Robert Lennox, and A. Louise Bradley \\ School of Physics, Trinity College Dublin, Dublin 2, Ireland \\ *Corresponding author: sylwester@eeng.dcu.ie ,Tel: +353 (0) 17005884
}

\begin{abstract}
A bulk semiconductor optical amplifier (SOA) utilizing a multi-contact biasing method is characterized as a function of the bias current supplied to each section Firstly, amplified spontaneous emission spectra and characteristic light-current profiles are analyzed. Single-pass gain and noise figure of the SOA are then assessed by injecting a continuous wave input beam. Finally, the amplification of a $10 \mathrm{~Gb} / \mathrm{s}\left(2^{15}-1\right)$ long pseudo random bit sequence signal is analysed. The obtained results, particularly at a bias condition of $90 \mathrm{~mA}-50 \mathrm{~mA}-10 \mathrm{~mA}$, reveal the potential of this device as a low noise figure in-line amplifier.
\end{abstract}

\section{INTRODUCTION}

Semiconductor optical amplifiers (SOAs) have been the subject of intense research owing to their nonlinear behaviour. Applications in all-optical communication systems as wavelength converters, logic gating, memory elements, packets forwarding, and others, have been demonstrated. The use of SOAs operating in a linear regime for amplification of optical signals in C-band communication systems seems to be limited because of the establishment of mature devices as Erbium doped fibre amplifiers (EDFA), which provide high gain and output saturation power, with a lower noise penalty. On the other hand, the recent demand for optical power budget extension in Giga-capable passive optical networks (GPON) has broadened the perspectives for SOAs as linear amplifiers (mainly in-line, but also as booster and pre-amplifiers) [1]-[2]. Indeed, they have a clear role to play in extending the distances currently covered by GPONs since upstream traffic may be positioned at $1300 \mathrm{~nm}$, where fibre-based amplifier technologies are not efficient [3]. In order to consolidate them as strong candidates for budget extension between the central office and end-customers, SOAs are required to provide both gain and low noise figure (NF) in the PON wavelength assignment of $1490 \mathrm{~nm}$ downstream and $1310 \mathrm{~nm}$ upstream. Such given wavelengths rule out EDFAs and place SOAs in a good position since the material composition of their typical InGaAsP active layer can be set to amplify signals in the desired range. Regarding the NF, it remains an issue for SOAs and there have been some efforts to reduce it such as the use of holding beams [4] or the increment of confinement factor in the active waveguide [5]. In this context, we aim to address the problem of NF improvement in bulk-SOAs by using a multi-electrode device. Firstly a typical characterization of collected amplified spontaneous emission (ASE) and single-pass gain is achieved for different bias conditions. Then the $\mathrm{NF}$ is calculated from the recorded data. We show that a bias configuration with small current (10 mA) at the output section and large current $(90 \mathrm{~mA})$ at the input section provides the lowest NF $(6 \mathrm{~dB})$ in the analysed SOA.

\section{EXPERIMENT}

The device under test is a three-electrode InGaAsP-InP bulk-SOA. Each section of the device is $250 \mu \mathrm{m}$ long, with a $750 \mu \mathrm{m}$ total length. The SOA is biased by using independent current sources $I_{1}$ to $I_{3}$ as depicted in the schematic of Fig. 1(a) and is temperature controlled at $21{ }^{\circ} \mathrm{C}$. Measurements of the amplified spontaneous emission (ASE) spectra and optical collected power versus bias current (LI) are performed initially. ASE measurements are recorded using an optical spectrum analyzer (OSA) for a number of combinations of bias current supplied to each section. It should be noted that according to the SOA manufacturer, the maximum current supplied to a single section cannot exceed $100 \mathrm{~mA}$ whereas the total bias is limited to $150 \mathrm{~mA}$ for temperature stability of the amplifier. Thus, ASE spectra at three different bias conditions are shown in Fig. 2(a). For the sake of simplicity, the bias notation is given in the order of input section first and output section last. For instance, a bias configuration of $90 \mathrm{~mA}-50 \mathrm{~mA}-10 \mathrm{~mA}$ indicates a bias current in the input section of $90 \mathrm{~mA}$ and so on. Moreover, in respect to the schematic diagram shown in Fig. 1(a), all reported measurements are obtained by collecting the ASE spectral density at the output (right facet) of the SOA. As it is observed from the ASE spectra in Fig. 2(a), the bias combination of $90 \mathrm{~mA}-50 \mathrm{~mA}-10 \mathrm{~mA}$ provides the lowest power level measured at the SOA right facet. This is because the small bias supplied to the output section is not enough to generate sufficient spontaneous emission nor to amplify light originating from the two other sections. 

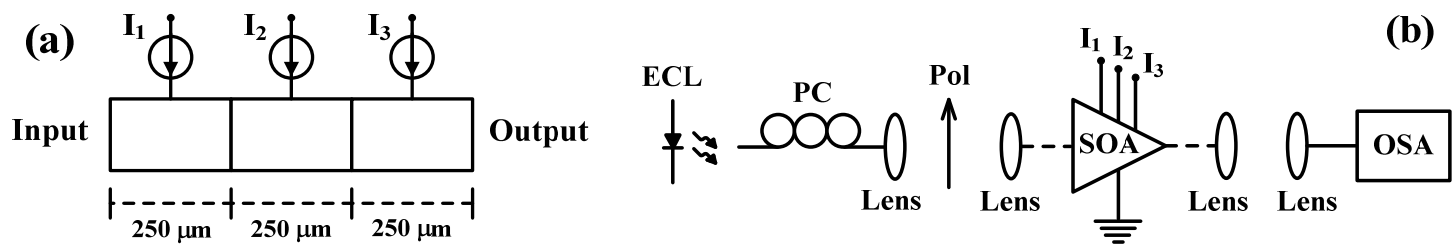

Fig. 1. (a) Schematic diagram of the multi-electrode SOA. (b) Experimental setup utilized for measuring the single-pass gain and noise figure.
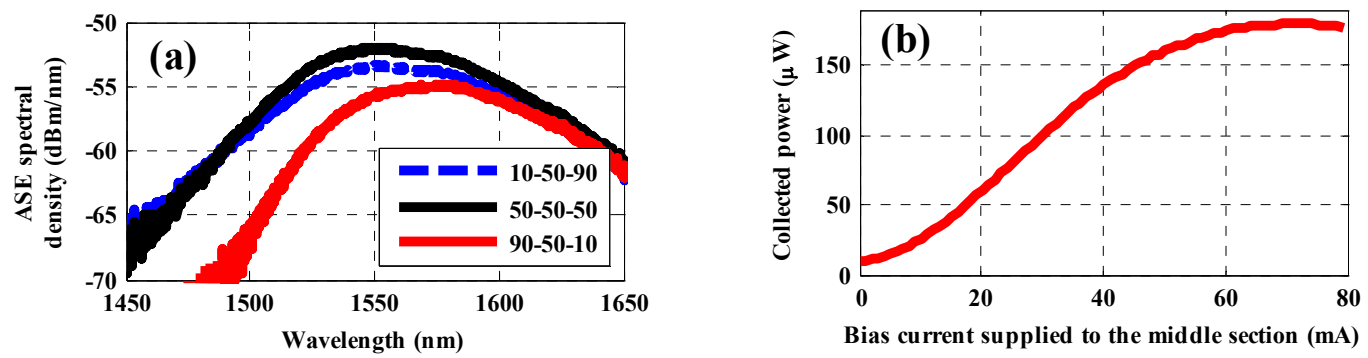

Fig. 2. (a) Collected ASE spectra for three different combinations of supplied bias. (b) LI curve showing optical collected power as a function of bias current supplied to middle section. The bias of the input and output section is kept constant at $33 \mathrm{~mA}$.

The influence of the bias current supplied to a given section on the total collected ASE is clearly observed from the LI response of the SOA depicted in Fig. 2(b). In this case, input and output sections are biased at $33 \mathrm{~mA}$, whilst the bias in the middle section is varied from 0 to $80 \mathrm{~mA}$. The overall bias condition approximates that of a standard SOA. The ASE power is measured by using a wide aperture integrating sphere and a power meter placed after the coupling lens at the SOA output facet.

In order to determine the single-pass gain and the noise figure, the multi-electrode SOA is characterized under continuous wave operation by using the experimental setup depicted in Fig. 1(b). An external cavity laser (ECL) with wavelength tuning from $1490 \mathrm{~nm}$ to $1590 \mathrm{~nm}$ and output power from $-20 \mathrm{dBm}$ to $+5 \mathrm{dBm}$ provides the input beam launched into the SOA. Injection and collection of light from the amplifier is performed in free space utilizing a 0.68 numerical aperture moulded aspheric lens and 3D flexure stage combination. SOA coupling losses are calculated by finding the geometrical modal mismatch between the circular mode profile of the collecting single mode fibre and the elliptical mode profile of the spontaneous emission at the amplifier facet. Injection and collection losses of $\sim 5.5 \mathrm{~dB}$ and $\sim 3.5 \mathrm{~dB}$ are estimated at the input and output facets, respectively. A polarization controller ( $\mathrm{PC}$ ) and polarizer $(\mathrm{Pol})$ allows aligning the state of polarization of the input beam to the transverse electrical (TE) propagation mode of the active waveguide. The output signal is collected using an OSA with a resolution bandwidth of $0.02 \mathrm{~nm}$. The aim of the experiment is to determine the optimum bias combination supplied to the SOA which results in the lowest noise figure. It is important to mention that the NF is calculated from the standard approach discussed in [6], with the signal spontaneous noise factor $F=2 \rho_{A S E} / G h v$ being the dominant contributor to the overall noise figure. In this equation, $\rho_{A S E}$ is the ASE spectral density in the same polarization state as the input and amplified optical signal, $G$ is the single-pass gain, $h$ is the Planck constant and $v$ is the linear frequency of the injected beam.

\section{RESULTS AND DISCUSSION}

Single-pass gain and noise figure of the multi-electrode device are characterised with respect to the injected beam wavelength and power for a number of different bias currents. The experimental results are shown in Fig. 3 for the injected beam polarized along the TE propagation mode of the active waveguide. In fact, a gain polarization sensitivity of $2 \mathrm{~dB}$ is measured. As observed from the plots depicted in Fig. 3(a) and (b), obtained for an input beam power of $-10 \mathrm{dBm}$, the single-pass gain is different for the three reported bias combinations. The largest gain is obtained for $50 \mathrm{~mA}-50 \mathrm{~mA}-50 \mathrm{~mA}$, exhibiting a maximum of $15 \mathrm{~dB}$ at $1560 \mathrm{~nm}$. Moreover, a minimum NF of $6 \mathrm{~dB}$ is obtained for both $50 \mathrm{~mA}-50 \mathrm{~mA}-50 \mathrm{~mA}$ and $90 \mathrm{~mA}-50 \mathrm{~mA}-10 \mathrm{~mA}$ bias configurations, whilst a clear increase of noise figure is observed for $10 \mathrm{~mA}-50 \mathrm{~mA}-90 \mathrm{~mA}$. On the other hand, as depicted in Fig. 3(c) and (d), the minimum NF as a function of the SOA output power is obtained for a bias of $90 \mathrm{~mA}-50 \mathrm{~mA}-$ 
$10 \mathrm{~mA}$ while the amplifier is operating in a linear regime (below the output saturation power of $\sim 5 \mathrm{dBm}$ ) and the input beam wavelength is $1575 \mathrm{~nm}$. Noise figure, single-pass gain and saturation characteristics of this device suggest that it would be well suited for application as an in-line amplifier at a bias condition of $90 \mathrm{~mA}-50 \mathrm{~mA}-$ $10 \mathrm{~mA}$. Indeed, for such a bias configuration, the carrier density profile along the active waveguide presents decreasing steep gradient, with the high bias at the input section being responsible for the overall low NF of the amplifier. Conversely, a higher bias at the output and lower bias at the input sections yields a higher noise figure and a higher saturation output power, as depicted in Fig. 3(c). To the best of our knowledge, noise figure measurements obtained in the present work are comparable to record values reported by using different approaches and active layer structures $(\mathrm{NF}=5 \mathrm{~dB}$ for a bulk-SOA by using a holding beam scheme [4], $\mathrm{NF}=3.5 \mathrm{~dB}$ for a MQW [5], and $\mathrm{NF}=4.8 \mathrm{~dB}$ for a gain-clamped amplifier [7]). As a matter of fact, the only work obtaining a NF of $5.5 \mathrm{~dB}$ on multi-electrode bulk-SOA is discussed in [8], even though no relevant NF plots are reported. On the other hand, wavelength at maximum gain can be changed to fit PON networks upstream and downstream specifications by changing active layer composition of the proposed multi-electrode device approach. Similarly, fibre-to-fibre single-pass gain can be substantially improved by integrating the bulk-SOA into a single chip.

It is worth discussing that the measured NFs depicted in Fig. 3(d) at bias conditions of 50mA-50mA-50mA and $90 \mathrm{~mA}-50-\mathrm{mA}-10 \mathrm{~mA}$ are the same for output powers around $6 \mathrm{dBm}$. This could be due to current leakage in the active waveguide as it is not separated into electrically isolated sections (even though the electrodes are electrically isolated). If this is the case then the difference in the gradient of the current supplied in these two cases does not differ enough to translate into an equivalent gradient in carrier density distribution. The only significant difference observed is when using diametrically opposed gradients, such as $90 \mathrm{~mA}-50 \mathrm{~mA}-10 \mathrm{~mA}$ and 10mA-50mA-90mA.

In order to obtain a better insight of the optimum bias configuration for low noise figure, the multi-electrode SOA under analysis is tested as a function of bias supplied to the middle section whilst input and output sections are biased at $90 \mathrm{~mA}$ and $10 \mathrm{~mA}$, respectively. The results are presented in Fig. 4. The noise figure decreases with increasing middle section bias, while the single-pass gain increases. The rise in single-pass gain can be attributed to an overall increment in the carrier density along the waveguide. A higher single-pass gain in turn contributes towards a lower NF.

Finally, the performance of the multi-electrode SOA as in-line amplifier is tested by using the same experimental setup as depicted in Fig. 1(b) but injecting a $10 \mathrm{~Gb} / \mathrm{s}\left(2^{15}-1\right)$ long non-return-to-zero (NRZ) pseudo random bit sequence (PRBS) at $1575 \mathrm{~nm}$ and average power of $-5 \mathrm{dBm}$. The current supplied to the SOA is set to $90 \mathrm{~mA}-50 \mathrm{~mA}-10 \mathrm{~mA}$. As shown in Fig. 5, the measured eye diagram reveals a relatively insignificant timing jitter degradation of the output signal (right) after propagation through the SOA with respect to the input signal (left), whilst preserving a minimum extinction ratio of $11.78 \mathrm{~dB}$.
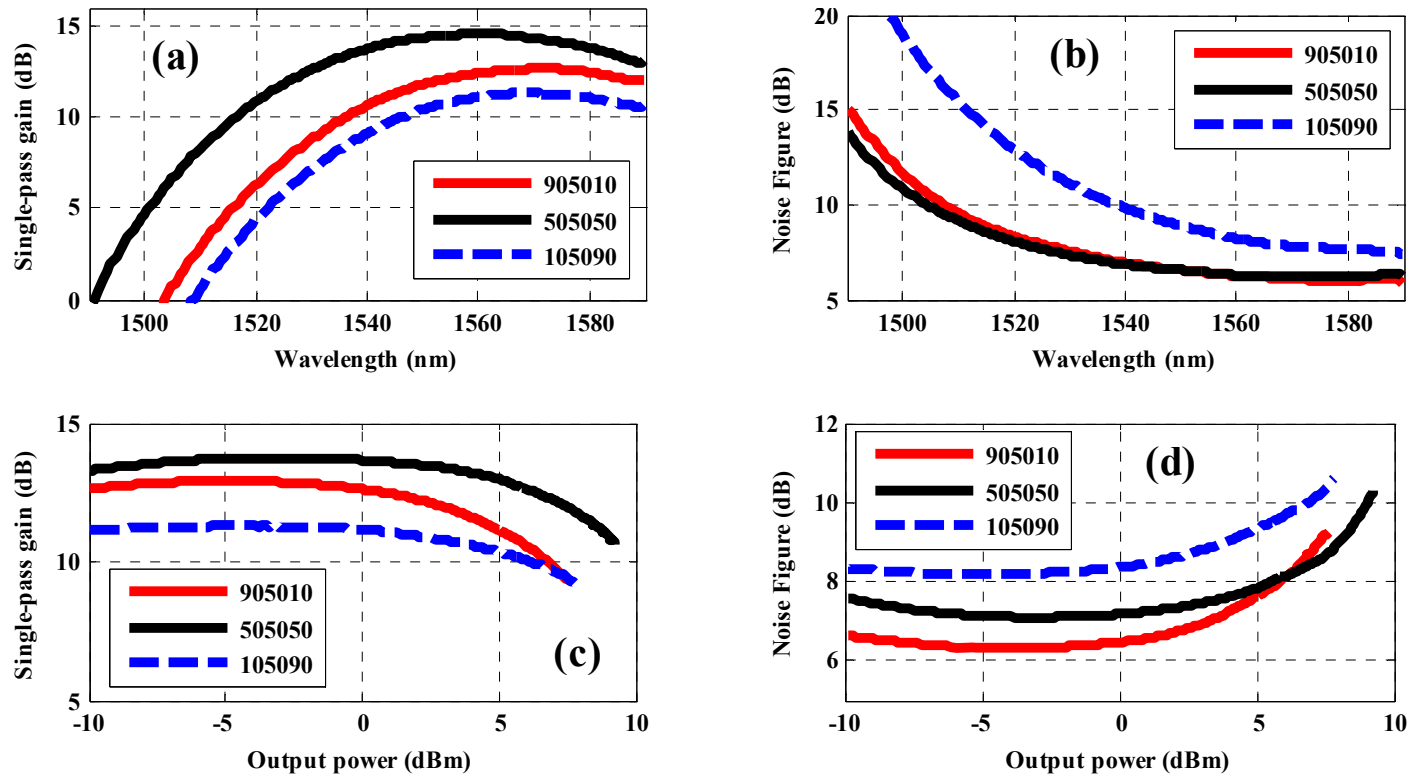

Fig. 3. Single-pass gain and noise figure in terms of the injected beam wavelength and SOA output power. In (a) and (b), an optical power of $-10 \mathrm{dBm}$ is injected. In (c) and (d), a beam wavelength of $1575 \mathrm{~nm}$ is considered. 


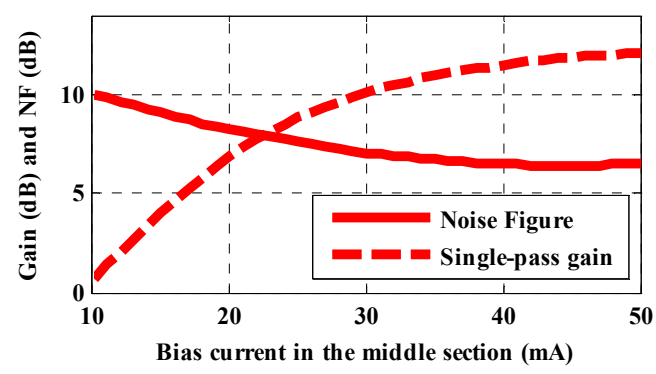

Fig. 4. Single-pass gain and noise figure as a function of the bias current supplied to the middle section. The injected signal power and wavelength are $-5 \mathrm{dBm}$ and $1580 \mathrm{~nm}$, respectively.
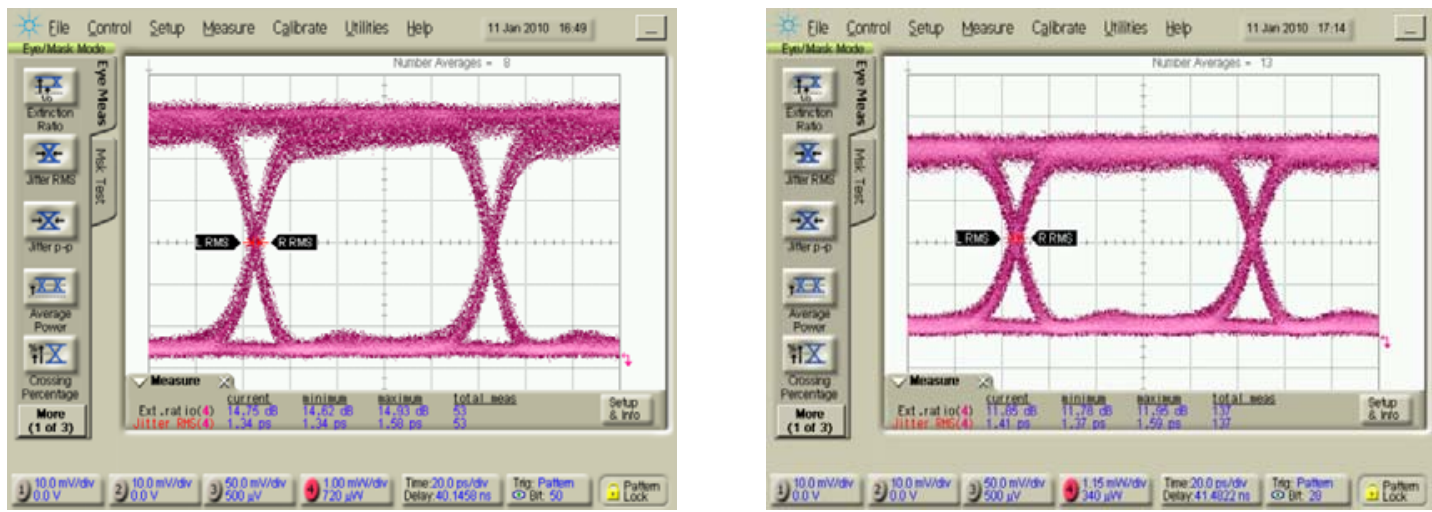

Fig. 5. Eye diagrams retrieved from an optical sampling scope illustrating the NRZ PRBS signal at the input (left) and output (right) of the multi-electrode SOA under test.

\section{ACKNOWLEDGMENTS}

The authors would like to thank Enterprise Ireland for its support under the Project TD/2009/0303 and the Higher Education Authority Program for Research in Third Level Institutions (2007-2011) via the INSPIRE Programme.

\section{REFERENCES}

[1] L. Spiekman, D. Piehler, P. Iannone, K. Reichmann, and H.-H. Lee. 'Semiconductor optical amplifiers for FTTx.' In Proc of ICTON 2007, Paper Mo.D2.4, 48-50, 2007.

[2] P. Chanclou, N. Genay, Z. Belfqih, F. Saliou, Q. Liu, and T. Soret. 'Invited paper: Next generation PON using amplification.’ In Proc of Photonics and Switching Topical Meeting, 101-102, 2007.

[3] C. Michie, A. E. Kelly, J. McGeough, S. Karagiannopoulos, and I. Andonovic. 'Optically amplified passive optical networks: a power budged analysis.' Journal of Optical Networking, 8(4), 370-382, 2009.

[4] A. Crottini, F. Salleras, P. Moreno, M.-A. Dupertuis, B. Deveaud, and R. Brenot. 'Noise figure improvement in semiconductor optical amplifiers by holding beam at transparency scheme.' Photon. Tech. Lett., 17(5), 977-979, 2005.

[5] K. Morito, S. Tanaka, S. Tomabechi, and A. Kuramata, 'A broadband MQW semiconductor optical amplifier with high saturation output power and low noise figure.' Photon. Tech. Lett., 17(5), 974-976, 2005.

[6] D. M. Baney, P. Gallion, and R.S. Tucker. 'Theory and measurement techniques for the noise figure of optical amplifiers.' Optical Fiber Technology, 6, 122-154, 2000.

[7] G. Guiliani, and D. D'Alessandro. 'Noise analysis of conventional and gain-clamped semiconductor optical amplifiers.' Journal of Lightwave Technology, 18(9), 1256-1263, 2000.

[8] S. S. Saini, J. Bowser, R. Enke, V, Luciani, P. J. S. Heim, and M. Dagenais. 'A semiconductor optical amplifier with high saturation power, low noise figure and low polarization dependent gain over the C-band'. In Proc of Lasers and ElectroOptics Society (LEOS), paper MK6, 102-103, 2004. 